PROCEEDINGS OF THE

AMERICAN MATHEMATICAL SOCIETY

Volume 132, Number 10, Pages 2849-2856

S 0002-9939(04)07072-8

Article electronically published on June 2, 2004

\title{
OFF-DIAGONAL SUBMATRICES OF A HERMITIAN MATRIX
}

\author{
CHI-KWONG LI AND YIU-TUNG POON
}

(Communicated by Joseph A. Ball)

\begin{abstract}
A necessary and sufficient condition is given to a $p \times q$ complex matrix $X$ to be an off-diagonal block of an $n \times n$ Hermitian matrix $C$ with prescribed eigenvalues (in terms of the eigenvalues of $C$ and singular values of $X)$. The proof depends on some recent breakthroughs in the study of spectral inequalities on the sum of Hermitian matrices by Klyachko and Fulton. Some interesting geometrical properties of the set $S$ of all such matrices are derived from the main result. These results improve earlier ones that only give partial information for the set $S$.
\end{abstract}

\section{INTRODUCTION}

Let $\mathcal{H}_{n}$ be the real linear space of $n \times n$ complex Hermitian matrices, and let $\mathcal{U}_{n}$ be the group of $n \times n$ unitary matrices. For $c=\left(c_{1}, \ldots, c_{n}\right) \in \mathbb{R}^{n}$ with $c_{1} \geq \cdots \geq c_{n}$, let $\mathcal{U}(c)$ be the set of matrices in $\mathcal{H}_{n}$ with eigenvalues $c_{1}, \ldots, c_{n}$. Equivalently, $\mathcal{U}(c)$ is the unitary (similarity) orbit of the diagonal matrix $\operatorname{diag}\left(c_{1}, \ldots, c_{n}\right)$. In this paper, a necessary and sufficient condition is given for a $p \times q$ complex matrix $X$ to be an off-diagonal block of a matrix $C \in \mathcal{U}(c)$ in terms of the singular values of $X$. The set of all such matrices $X$ can be viewed as the projection of $\mathcal{U}(c)$.

Let $p$ and $q$ be positive integers such that $p+q \leq n$. Consider the set

$$
S_{p, q}(c)=\left\{A_{13} \in \mathbb{C}^{p \times q}: \text { there exists } A=\left(A_{i j}\right)_{1 \leq i, j \leq 3} \in \mathcal{U}(c)\right\} .
$$

Clearly, in the block matrix $A=\left(A_{i j}\right)_{1 \leq i, j \leq 3}$ above, $A_{11} \in \mathcal{H}_{p}$ and $A_{33} \in \mathcal{H}_{q}$. In statistics and other applications, there is considerable interest (see [1, [5], [7, [21] and their references) in studying inequalities relating the singular values of matrices in $S_{p, n-p}(c)$ and $c$. In 21], the authors proved that if $p \leq n / 2$ and $X \in S_{p, n-p}(c)$ has singular values $s_{1} \geq \cdots \geq s_{p}$, then

$$
\frac{1}{2} \sum_{j=1}^{m}\left(c_{j}-c_{n-j+1}\right) \geq \sum_{j=1}^{m} s_{j}, \quad m=1, \ldots, p .
$$

Using this result and the theory on Schur convex functions, one can (see 21]) refine and shorten the proofs of many existing inequalities. Evidently, the above result

Received by the editors February 18, 2002 and, in revised form, October 24, 2002.

2000 Mathematics Subject Classification. Primary 15A18, 15A42.

Key words and phrases. Hermitian matrices, singular values, eigenvalues, LittlewoodRichardson rules.

The first author's research was partially supported by an NSF grant. 
on $S_{p, n-p}(c)$ is equivalent to

$$
S_{p, n-p}(c) \subseteq\left\{X \in \mathbb{C}^{p \times(n-p):}: \begin{array}{l}
X \text { has singular values } \\
s_{1} \geq \cdots \geq s_{p} \text { satisfying (1) }
\end{array}\right\} .
$$

In [5] the authors studied some convexity features of $\mathcal{U}(c)$, and showed that the equality in (2) holds if and only if $S_{p, n-p}(c)$ is convex, and this happens if and only if $\left(c_{1}, \ldots, c_{p}\right)$ and $\left(c_{n-p+1}, \ldots, c_{n}\right)$ are arithmetic progressions with the same common difference.

In this paper, we give a complete description of $S_{p, q}(c)$ for general $(p, q)$. In particular, it follows from our result that the set $S_{p, q}(c)$ is always star-shaped with the zero matrix as a star center; see Corollary 2.4. Some examples and remarks related to our results are also given.

There are two key ingredients in our proofs. First, we need the recent major breakthrough in the study of spectral inequalities on the sum of Hermitian matrices by Klyachko [17] and its generalization by Fulton [11]; see also [10] for an excellent survey. Second, we need some matrix techniques to relate the results on the sum of Hermitian matrices to the off-diagonal blocks of a Hermitian matrix.

Although our discussion concerns complex Hermitian matrices, all our results are valid (with the same proofs) for real symmetric matrices and Hermitian matrices over real quaternions.

In our discussion, the sets of eigenvalues or singular values always mean the multi-sets of eigenvalues or singular values counting multiplicities.

We thank Professor W. Fulton for some helpful comments on an early draft of this paper.

\section{MAin RESUlt}

As mentioned in the Introduction, there are two important ingredients in our proofs. We first present a result of Fulton 11 concerning the sum of Hermitian matrices. This result is a generalization of a remarkable result of Klyachko [17] on spectral inequalities on the sum of Hermitian matrices.

Let $I=\left(i_{1}, \ldots, i_{m}\right)$ be an increasing subsequence of $[n]=(1, \ldots, n)$. Define

$$
\lambda(I)=\left(i_{m}-m, \ldots, i_{1}-1\right) .
$$

Suppose $J=\left(j_{1}, \ldots, j_{m}\right)$ and $K=\left(k_{1}, \ldots, k_{m}\right)$ are also increasing subsequences of $[n]$. We say that $(I, J, K) \in L R_{m}^{n}$ if the Littlewood-Richardson coefficient $c_{\lambda(I) \lambda(J)}^{\lambda(K)}$ of the three partitions $\lambda(I), \lambda(J)$, and $\lambda(K)$ is equal to one; see [9]. Our proofs depend on the following result in 11]; see Theorem 1, the discussion after the statement of Theorem 2, and the proofs in Section 2 of that paper.

Lemma 2.1. There exist Hermitian matrices $A, B, C$ with eigenvalues

$$
a_{1} \geq \cdots \geq a_{n}, \quad b_{1} \geq \cdots \geq b_{n}, \quad \text { and } \quad c_{1} \geq \cdots \geq c_{n},
$$

respectively, such that

$$
A+B \geq C, \quad \text { i.e., } A+B-C \text { is positive semidefinite }
$$

if and only if for each $m \in\{1, \ldots, n\}$ and $(I, J, K) \in L R_{m}^{n}$,

$$
\sum_{i \in I} a_{i}+\sum_{j \in J} b_{j} \geq \sum_{k \in K} c_{k}
$$


Moreover, if there is an $m \in\{1, \ldots, n\}$ such that the equality in (4) holds for some $(I, J, K) \in L R_{m}^{n}$, then there exist $A_{1}, B_{1}, C_{1} \in \mathcal{H}_{m}$ with sets of eigenvalues (counting multiplicities) $\left\{a_{i}: i \in I\right\},\left\{b_{j}: j \in J\right\},\left\{c_{k}: k \in K\right\}$, respectively, so that $A_{1}+B_{1}=C_{1}$, and in the case of $n>m$, there exist $A_{2}, B_{2}, C_{2} \in \mathcal{H}_{n-m}$ with sets of eigenvalues (counting multiplicities) $\left\{a_{i}: i \in[n] \backslash I\right\},\left\{b_{j}: j \in[n] \backslash J\right\}$, $\left\{c_{k}: k \in[n] \backslash K\right\}$, respectively, so that $A_{2}+B_{2} \geq C_{2}$.

Next, we establish a lemma relating a Hermitian matrix sum to the off-diagonal block of a Hermitian matrix.

Lemma 2.2. Suppose $a_{1} \geq \cdots \geq a_{n}, b_{1} \geq \cdots \geq b_{n}, c_{1} \geq \cdots \geq c_{n}$, and $t \in$ $[0,1]$ are such that $\sum_{j=1}^{n} c_{n}=t\left\{\sum_{j=1}^{n}\left(a_{j}+b_{j}\right)\right\}$ and for each $m \in\{1, \ldots, n\}$ and $(I, J, K) \in L R_{m}^{n}$,

$$
t\left\{\sum_{i \in I} a_{i}+\sum_{j \in J} b_{j}\right\} \geq \sum_{k \in K} c_{k} .
$$

Then there exist $A, B, C \in \mathcal{H}_{n}$ with eigenvalues $a_{1} \geq \cdots \geq a_{n}, b_{1} \geq \cdots \geq b_{n}$, $c_{1} \geq \cdots \geq c_{n}$, respectively, such that

$$
C=t(A+B) .
$$

Moreover, there exists a unitary matrix $U \in \mathcal{U}_{2 n}$ such that

$$
U^{*}\left(\begin{array}{cc}
A & 0 \\
0 & -B
\end{array}\right) U=\left(\begin{array}{cc}
* & C / 2 \\
C / 2 & *
\end{array}\right) \text {. }
$$

Proof. The first assertion follows from Lemma 2.1 To prove the second assertion, let $\theta \in[0, \pi / 4]$ be such that $\sin 2 \theta=t$, and let

$$
U=\left(\begin{array}{cc}
\cos \theta I_{n} & \sin \theta I_{n} \\
-\sin \theta I_{n} & \cos \theta I_{n}
\end{array}\right)
$$

Then

$$
U^{*}\left(\begin{array}{cc}
A & 0 \\
0 & -B
\end{array}\right) U=\left(\begin{array}{cc}
* & (A+B) \sin 2 \theta / 2 \\
(A+B) \sin 2 \theta / 2 & *
\end{array}\right)
$$

We are ready to give the characterization of

$$
S_{p, q}(c)=\left\{A_{13} \in \mathbb{C}^{p \times q}: \text { there exists } A=\left(A_{i j}\right)_{1 \leq i, j \leq 3} \in \mathcal{U}(c)\right\},
$$

where $p$ and $q$ are positive integers such that $p+q \leq n$. Since $X \in S_{p, q}(c)$ if and only if $X^{*} \in S_{q, p}(c)$, we only need to consider the case when $p \leq q$.

Theorem 2.3. Let $c_{1} \geq \cdots \geq c_{n}$. Suppose $p$ and $q$ are positive integers such that $p \leq q$ and $p+q \leq n$, and $X \in \mathbb{C}^{p \times q}$ has singular values $s_{1} \geq \cdots \geq s_{p}$. The following conditions are equivalent.

(a) $X \in S_{p, q}(c)$.

(b) For each $m \in\{1, \ldots, p\}$ and $(I, J, K) \in L R_{m}^{p}$,

$$
\sum_{i \in I} c_{i}-\sum_{j \in J} c_{n-j+1} \geq 2 \sum_{k \in K} s_{k}
$$

(c) There exist $A, B, S \in \mathcal{H}_{p}$ with eigenvalues $c_{1} \geq \cdots \geq c_{p}, c_{n-p+1} \geq \cdots \geq c_{n}$, and $s_{1} \geq \cdots \geq s_{p}$, respectively, such that $A-B \geq 2 S$. 
Proof. The equivalence of (b) and (c) follows from Lemma 2.1.

(a) $\Rightarrow$ (b): Suppose (a) holds, i.e., there is a matrix $A=\left(A_{i j}\right)_{1 \leq i, j \leq 3} \in \mathcal{U}(c)$ such that $A_{13}=X$. Then $\tilde{A}=-\left(-I_{p} \oplus I_{n-p}\right) A\left(-I_{p} \oplus I_{n-p}\right)$ has eigenvalues $-c_{n} \geq \cdots \geq-c_{1}$. Suppose the matrix

$$
A+\tilde{A}=2\left(\begin{array}{ccc}
0 & A_{12} & A_{13} \\
A_{12}^{*} & 0 & 0 \\
A_{13}^{*} & 0 & 0
\end{array}\right)
$$

has eigenvalues $2 \tilde{s}_{1} \geq \cdots \geq 2 \tilde{s}_{p} \geq \overbrace{0 \geq \cdots \geq 0}^{n-2 p} \geq-2 \tilde{s}_{p} \geq \cdots \geq-2 \tilde{s}_{1}$. By Lemma 2.1, condition (b) holds with $s_{j}$ replaced by $\tilde{s}_{j}$ for $j=1, \ldots, p$. Since $2\left(\begin{array}{cc}0 & A_{13}^{*} \\ A_{13}^{*} & 0\end{array}\right)$ is a principal submatrix of $A+\tilde{A}$ and has eigenvalues

$$
2 s_{1} \geq \cdots \geq 2 s_{p} \geq \overbrace{0 \geq \cdots \geq 0}^{q-p} \geq-2 s_{p} \geq \cdots \geq-2 s_{1},
$$

we have $\tilde{s}_{j} \geq s_{j}$ for $j=1, \ldots, p$, by the result in [8] (see also [15, Theorem 4.3.15]). Thus, condition (b) holds.

(b) $\Rightarrow$ (a): Suppose (b) holds. It suffices to construct a matrix

$$
A=\left(\begin{array}{ccc}
A_{11} & 0 & S \\
0 & A_{22} & 0 \\
S^{*} & 0 & A_{33}
\end{array}\right) \in \mathcal{U}(c)
$$

such that $S \in \mathbb{C}^{p \times q}$ has singular values $s_{1} \geq \cdots \geq s_{p}$. Then there exist $V_{1} \in \mathcal{U}_{p}$ and $V_{2} \in \mathcal{U}_{q}$ such that $V_{1}^{*} S V_{2}=X$ is the $(1,3)$ block of the matrix

$$
\left(V_{1} \oplus I_{n-p-q} \oplus V_{2}\right)^{*} A\left(V_{1} \oplus I_{n-p-q} \oplus V_{2}\right) \in \mathcal{U}(c) .
$$

To construct the matrix in (5), we first consider the case when $n=2 p$. We prove the result by induction on $p$. If $p=1$, then $t\left(c_{1}-c_{2}\right)=2 s_{1}$ for some $t \in[0,1]$. The result follows from Lemma 2.2. Suppose $p>1$, and the implication holds for lower dimensions. Let $t \in[0,1]$ be such that for each $m \in\{1, \ldots, p\}$ and $(I, J, K) \in L R_{m}^{p}$,

$$
t\left\{\sum_{i \in I} c_{i}-\sum_{j \in J} c_{n-j+1}\right\} \geq 2 \sum_{k \in K} s_{k},
$$

and the equality occurs at $(I, J, K) \in L R_{m^{\prime}}^{p}$ for some $m^{\prime} \in\{1, \ldots, p\}$. If $m^{\prime}=p$, condition (a) follows from Lemma 2.2. If $m^{\prime}<p$, then by Lemma 2.1 there exist $A_{1}, B_{1}, S_{1} \in \mathcal{H}_{m^{\prime}}$ with sets of eigenvalues $\left\{c_{i}: i \in I\right\},\left\{-c_{n-j+1}: j \in J\right\}$, and $\left\{s_{k}: k \in K\right\}$ respectively, satisfying $t\left(A_{1}+B_{1}\right)=2 S_{1}$, and there exist $A_{2}, B_{2}, S_{2} \in$ $\mathcal{H}_{p-m^{\prime}}$ with sets of eigenvalues $\left\{c_{i}: i \in[p] \backslash I\right\},\left\{-c_{n-j+1}: j \in[p] \backslash J\right\}$, and $\left\{s_{k}: k \in[p] \backslash K\right\}$ respectively, satisfying $t\left(A_{2}+B_{2}\right) \geq 2 S_{2}$. Consequently, by Lemma 2.2, there exists $U_{1} \in \mathcal{U}_{2 m^{\prime}}$ such that

$$
U_{1}^{*}\left(\begin{array}{cc}
A_{1} & 0 \\
0 & -B_{1}
\end{array}\right) U_{1}=\left(\begin{array}{cc}
* & S_{1} \\
S_{1}^{*} & *
\end{array}\right) .
$$

Moreover, by Lemma 2.1 again, the eigenvalues of $A_{2}, B_{2}, S_{2}$ satisfy the inductive hypothesis of the implication (b) $\Rightarrow(\mathrm{a})$. So, there exists $U_{2} \in \mathcal{U}_{2\left(p-m^{\prime}\right)}$ such that

$$
U_{2}^{*}\left(\begin{array}{cc}
A_{2} & 0 \\
0 & -B_{2}
\end{array}\right) U_{2}=\left(\begin{array}{cc}
* & S_{2} \\
S_{2}^{*} & *
\end{array}\right) .
$$


Let $A=A_{1} \oplus A_{2}, B=B_{1} \oplus B_{2}$ and $S=S_{1} \oplus S_{2}$. By a block permutation similarity, there exists $U_{3} \in \mathcal{U}_{2 p}$ such that

$$
U_{3}^{*}\left(\begin{array}{cc}
A & 0 \\
0 & -B
\end{array}\right) U_{3}=\left(\begin{array}{cc}
P & S \\
S^{*} & Q
\end{array}\right)
$$

which has eigenvalues $c_{1}, \ldots, c_{n}$.

Now, suppose $n>2 p$. Let

$$
D=\operatorname{diag}\left(c_{1}, \ldots, c_{p}, c_{n-p+1}, \ldots, c_{n}\right) .
$$

By the above construction, we can get a unitary matrix $U \in \mathcal{U}_{2 p}$ such that

$$
U^{*} D U=\left(\begin{array}{cc}
P & S \\
S^{*} & Q
\end{array}\right)
$$

where $S$ is $p \times p$ with singular values $s_{1} \geq \cdots \geq s_{p}$. If $D_{0}=\operatorname{diag}\left(c_{p+1}, \ldots, c_{n-p}\right)$, then $U^{*} D U \oplus D_{0}$ is permutationally similar to

$$
\left(\begin{array}{ccc}
P & 0 & S \\
0 & D_{0} & 0 \\
S^{*} & 0 & Q
\end{array}\right),
$$

which is the desired matrix satisfying (5) after we redefine the block sizes.

By the result in [5], the set $S_{p, q}(c)$ may not be convex; $S_{p, q}(c)$ is convex if and only if for $\ell=\min \{p, q\}$, the sequences $\left(c_{1}, \ldots, c_{\ell}\right)$ and $\left(c_{n-\ell+1}, \ldots, c_{n}\right)$ are in arithmetic progression with the same common difference. In general, we have the following.

Corollary 2.4. Let $c_{1} \geq \cdots \geq c_{n}$ and let $p, q$ be positive integers such that $p+q \leq$ $n$. Suppose $X \in S_{p, q}(c)$ has singular values $s_{1} \geq \cdots \geq s_{\ell}$ where $\ell=\min \{p, q\}$. If $Y \in \mathbb{C}^{p \times q}$ has singular values $t_{1}, \ldots, t_{\ell}$ satisfying $t_{i} \leq s_{i}$ for all $i=1, \ldots, \ell$, then $Y \in S_{p, q}(c)$. Equivalently, $R X S \in S_{p, q}(c)$ for all contractions $R, S$ of sizes $p$ and $q$, respectively. In particular, $t X \in S_{p, q}(c)$ for all $0 \leq t \leq 1$, i.e., $S_{p, q}(c)$ is star-shaped with the zero matrix as a star center.

Proof. Since $X \in S_{p, q}(c)$ if and only if $X^{*} \in S_{q, p}(c)$, we consider only the case $\ell=p$. If $X \in S_{p, q}(c)$ has singular values $s_{1} \geq \cdots \geq s_{p}$, then Theorem 2.3(b) holds. Since $t_{i} \leq s_{i}$ for all $i=1, \ldots, p$, (b) also holds with $s_{i}$ replaced by $t_{i}$. Hence, $Y \in S_{p, q}(c)$. Note that a matrix $Y \in \mathbb{C}^{p \times q}$ has singular values $t_{i} \leq s_{i}$, for all $i=1, \ldots, p$ if and only if $Y=R X S$ for some contractions $R, S$ of sizes $p$ and $q$, respectively. Therefore, the equivalent statement follows. The last assertion is clear.

\section{Remarks and examples}

It is interesting that by Corollary 2.4, if $X \in S_{p, q}(c)$, one can conclude without any additional information about $c$ that $R X S \in S_{p, q}(c)$ for any contractions $R$ and $S$ of sizes $p$ and $q$, respectively. In other words, if $A=\left(A_{i j}\right)_{i, j=1}^{3} \in \mathcal{U}(A)$ so that $A_{13} \in \mathbb{C}^{p \times q}$, then there is $\tilde{A}=\left(\tilde{A}_{i j}\right)_{i, j=1}^{3} \in \mathcal{U}(A)$ with $\tilde{A}_{i j}=R A_{13} S$. Note that in general the matrix $T=R^{*} \oplus I_{n-p-q} \oplus S$ is not unitary, and therefore one cannot simply let $\tilde{A}$ be $T^{*} A T$ although its $(1,3)$ block has the form $R A_{13} S$. Actually, in computation, even constructing $\tilde{A} \in \mathcal{U}(c)$ such that $\tilde{A}_{13}=A_{13} / 2$ for a given $A_{13} \in S_{p, q}(c)$ is a nontrivial problem. 
In the proof of the implication (a) $\Rightarrow(\mathrm{b})$ in Theorem 2.3, one actually has

$$
\sum_{i \in I} c_{i}-\sum_{j \in J} c_{n-j+1} \geq 2 \sum_{k \in K} s_{k}
$$

for any $(I, J, K) \in L R_{m}^{n}$, whereas our necessary and sufficient conditions only require those inequalities associated with $(I, J, K) \in L R_{m}^{p}$. Thus many inequalities associated with $(I, J, K) \in L R_{m}^{n}$ are redundant.

In [21], using the inequalities (11), which is a subcollection of those in Theorem 2.3)(b), and the theory of Schur convex functions, the authors refine and shorten the proofs of results on matrix inequalities relating the eigenvalues of $C \in \mathcal{H}_{n}$ and the singular values of an off-diagonal submatrix of $C$. Using the whole set of inequalities in Theorem 2.3 (b), one should be able to get more information. For example, for any $1 \leq i_{1}<\cdots<i_{\ell} \leq p$, we have

$$
\sum_{r=1}^{m}\left(c_{i_{r}}-c_{n-r+1}\right) \geq 2 \sum_{r=1}^{m} s_{i_{r}}, \quad m=1, \ldots, \ell .
$$

Thus, for any increasing convex function $f: \mathbb{R} \rightarrow \mathbb{R}$, we have

$$
\sum_{r=1}^{\ell} f\left(c_{i_{r}}-c_{n-r+1}\right) \geq \sum_{r=1}^{\ell} f\left(2 s_{i_{r}}\right) .
$$

In our main result and the supporting lemmas, we may replace $L R_{m}^{n}$ (or $L R_{m}^{p}$ ) by other sets. For example, we can use the set $\mathcal{L} R_{m}^{n}$ : the set of $(I, J, K)$ sequence triples such that the Littlewood-Richardson coefficient corresponding to the partitions $\lambda(I), \lambda(J), \lambda(K)$ is positive. As shown in [10 Theorem 18], these sequence triples $(I, J, K)$ are the same as the Horn-Thompson consistent sequences, which can be generated systematically as follows; see [6], 10], [14] and their references.

Let $I=\left(i_{1}, \ldots, i_{m}\right), J=\left(j_{1}, \ldots, j_{m}\right), K=\left(k_{1}, \ldots, k_{m}\right)$ be increasing subsequences of $[n]$.

1. For $m \geq 1, \sum_{\ell=1}^{m} k_{\ell}+m(m+1) / 2=\sum_{\ell=1}^{m}\left(i_{\ell}+j_{\ell}\right)$.

2. If $m>1$, then for any consistent triple $(U, V, W)$, where $U=\left(u_{1}, \ldots, u_{m^{\prime}}\right)$, $V=\left(v_{1}, \ldots, v_{m^{\prime}}\right)$ and $W=\left(w_{1}, \ldots, w_{m^{\prime}}\right)$ with $m^{\prime} \in\{1, \ldots, m-1\}$, we have

$$
\sum_{\ell=1}^{m^{\prime}} k_{w_{\ell}}+m^{\prime}\left(m^{\prime}+1\right) / 2 \geq \sum_{\ell=1}^{m^{\prime}}\left(i_{u_{\ell}}+j_{v_{\ell}}\right) .
$$

Although $\mathcal{L} R_{m}^{n}$ contains some redundant inequalities, it is relatively easy to generate them because one need not verify that the Littlewood-Richardson coefficient of $\lambda(I), \lambda(J)$, and $\lambda(K)$ is one. Here we give a short explanation of why $L R_{m}^{n}$ can be replaced by $\mathcal{L} R_{m}^{n}$.

It is known [20] (see also [18, [19]) that $a_{1} \geq \cdots \geq a_{n}, b_{1} \geq \cdots \geq b_{n}$, and $c_{1} \geq \cdots \geq c_{n}$ satisfy (4) for each $m \in\{1, \ldots, n\}$ and $(I, J, K) \in L R_{m}^{n}$ if and only if they satisfy (4) for each $m \in\{1, \ldots, n\}$ and $(I, J, K) \in \mathcal{L} R_{m}^{n}$. By the minimality of $L R_{m}^{n}$, if equality holds for some consistent triples $(I, J, K) \in \mathcal{L} R_{m}^{n}$, then we can find a triple $\left(I^{\prime}, J^{\prime}, K^{\prime}\right) \in L R_{m^{\prime}}^{n}$ for which the equality holds. In fact, if equality holds in (4) for some $(I, J, K) \in \mathcal{L} R_{m}^{n}$ with $m \in\{1, \ldots, n\}$, but

$$
\sum_{i \in I^{\prime}} a_{i}+\sum_{j \in J^{\prime}} b_{j}>\sum_{k \in K^{\prime}} c_{k}
$$


for all $\left(I^{\prime}, J^{\prime}, K^{\prime}\right) \in L R_{m^{\prime}}^{n}$ with $m^{\prime} \in\{1, \ldots, n\}$, then there exists $d>0$ such that

$$
\sum_{i \in I^{\prime}}\left(a_{i}-d\right)+\sum_{j \in J^{\prime}}\left(b_{j}-d\right)>\sum_{k \in K^{\prime}} c_{k}
$$

for all $\left(I^{\prime}, J^{\prime}, K^{\prime}\right) \in L R_{m^{\prime}}^{n}$ with $m^{\prime} \in\{1, \ldots, n\}$, which implies (by the minimality of $L R_{m}^{n}$ ) that

$$
\sum_{k \in K} c_{k}=\sum_{i \in J} a_{i}+\sum_{j \in J} b_{j} \leq \sum_{i \in I}\left(a_{i}-d\right)+\sum_{j \in J}\left(b_{j}-d\right)
$$

a contradiction.

In general, condition (b) in Theorem 2.3 is difficult to check. If $c_{1}, \ldots, c_{n}$ have some special properties, the situations are more pleasant.

Examples. Let $p$ and $q$ be positive integers such that $p \leq q$ and $p+q \leq n$.

(i) If $\left(c_{1}, \ldots, c_{p}\right)$ and $\left(c_{n-p+1}, \ldots, c_{n}\right)$ are arithmetic progressions with the same common difference, then condition (b) reduces to

$$
\sum_{j=1}^{r}\left(c_{j}-c_{n-j+1}\right) \geq \sum_{j=1}^{r} 2 s_{j}, \quad r=1, \ldots, p .
$$

(ii) If $c_{1}=\cdots=c_{p}$ or $c_{n-p+1}=\cdots=c_{n}$, then (b) reduces to

$$
c_{j}-c_{n-j+1} \geq 2 s_{j} \quad \text { for each } j \in\{1, \ldots, p\} .
$$

For the verification of (i), see [5]. To verify (ii), we focus on the case when $c_{n-p+1}=\cdots=c_{n}$. First, note that (6) are just the subcollection of inequalities in Theorem 2.3(b) with $|I|=|J|=|K|=1$ and $J=\{1\}$. Now, suppose (6) holds. Then for each $j \in\{1, \ldots, p\}$, one can construct a Hermitian matrix of the form

$$
C_{j}=\left(\begin{array}{cc}
r_{j} & s_{j} \\
s_{j} & t_{j}
\end{array}\right)
$$

such that $r_{j} t_{j}-s_{j}^{2}=c_{j} c_{n}$ and $r_{j}+s_{j}=c_{j}+c_{n}$, and thus $C_{j}$ has eigenvalues $c_{j}, c_{n}$. Now, a permutation similarity of the matrix

$$
C_{1} \oplus \cdots \oplus C_{p} \oplus \operatorname{diag}\left(c_{p+1}, \ldots, c_{n-p}\right)
$$

will yield $A=\left(A_{i j}\right)_{i, j=1}^{3} \in \mathcal{U}(c)$ such that $A_{13} \in \mathbb{C}^{p \times q}$ has singular values $s_{1}, \ldots, s_{p}$. By the implication (a) $\Rightarrow$ (b) in Theorem 2.3, we see that all the inequalities in (b) hold.

\section{REFERENCES}

[1] T. Ando, Bloomfield-Watson-Knott type inequalities for eigenvalues, Taiwanese J. Math. 5 (2001), 443-469. MR 2002f:15022

[2] R. Bhatia, Matrix Analysis, Springer-Verlag, New York, 1996. MR 98i:15003

[3] A.S. Buch, The saturation conjecture (after A. Knutson and T. Tao). With an appendix by William Fulton. Enseign. Math. (2) 46 (2000), no. 1-2, 43-60. MR 2001g:05105

[4] M. Cho and M. Takaguchi, Some classes of commuting $m$-tuples of operators, Studia Math. 80 (1984), 245-259. MR 86g:47059

[5] M.D. Choi, C.K. Li and Y.T. Poon, Some convexity features associated with unitary orbits, Canad. J. Math. 55 (2003), 91-111. MR 2003m:15051

[6] J. Day, W. So and R.C. Thompson, The spectrum of a Hermitian matrix sum, Linear Algebra Appl. 280 (1998), 289-332. MR 99f:15009 
[7] S.W. Drury, S. Liu, C.-Y. Lu, S. Puntanen, and G.P.H. Styan, Some comments on several matrix inequalities with applications to canonical correlations, to appear in the Special Issue of Sankhya associated with "An International Conference in Honor of Professor C.R. Rao on the occasion of his 80th Birthday, Statistics: Reflections on the Past and Visions for the Future, The University of Texas at San Antonio, March 2000".

[8] K. Fan and G. Pall, Imbedding conditions for Hermitian and normal matrices, Canad. J. Math. 9 (1957), 298-304. MR 19:6e

[9] W. Fulton, Young Tableaux, Cambridge University Press, Cambridge, 1997. MR 99f:05119

[10] W. Fulton, Eigenvalues, invariant factors, highest weights, and Schubert calculus. Bull. Amer. Math. Soc. (N.S.) 37 (2000), 209-249. MR 2001g:15023

[11] W. Fulton, Eigenvalues of majorized Hermitian matrices, and Littlewood-Richardson coefficients, Linear Algebra Appl. 319 (2000), 23-36. MR 2002a:15024

[12] U. Helmke and J. Rosenthal, Eigenvalue inequalities and Schubert calculus, Math. Nachr. 171 (1995), 207-225. MR 96b:15039

[13] A. Horn, Doubly stochastic matrices and the diagonal of a rotation matrix, Amer. J. Math. 76 (1954), 620-630. MR 16:105c

[14] A. Horn, Eigenvalues of sums of Hermitian matrices, Pacific J. Math. 12 (1962), 225-241. MR 25:3941

[15] R.A. Horn and C.R. Johnson, Matrix Analysis, Cambridge University Press, Cambridge, 1985. MR 87e:15001

[16] R.A. Horn and C.R. Johnson, Topics in Matrix Analysis, Cambridge University Press, Cambridge, 1991. MR 92e:15003

[17] A.A. Klyachko, Stable bundles, representation theory and Hermitian operators, Selecta Math. (N.S.) 4 (1998), 419-445. MR 2000b:14054

[18] A. Knutson and T. Tao, The honeycomb model of $\mathrm{GL}_{n}(c)$ tensor products. I. Proof of the saturation conjecture, J. Amer. Math. Soc. 12 (1999), no. 4, 1055-1090. MR 2000c:20066

[19] A. Knutson and T. Tao, Honeycombs and sums of Hermitian matrices, Notices of the Amer. Math. Soc. 48 (2001), no. 2, 175-186. MR 2002g:15020

[20] A. Knutson, T. Tao, and C. Woodward, Honeycombs II: Facets of the Littlewood-Richardson cone, to appear.

[21] C.K. Li and R. Mathias, Inequalities on the singular values of an off-diagonal block of a Hermitian matrix, J. of Inequalities and Applications 3 (1999), 137-142. MR 2001b:15025

[22] R.C. Thompson and L.J. Freede, Eigenvalues of partitioned Hermitian matrices, Bulletin Austral. Math. Soc. 3 (1970), 23-37. MR 42:286

[23] A. Zelevinsky, Littlewood-Richardson semigroups, MSRI preprint 1997-044.

Department of Mathematics, College of William and Mary, P.O. Box 8795, WilliamsBURG, VIRGINIA 23187-8795

E-mail address: ckli@math.wm.edu

Department of Mathematics, Iowa State University, Ames, Iowa 50011

E-mail address: ytpoon@iastate.edu 